\title{
Combining synchronous averaging with a Gaussian mixture model novelty detection scheme for vibration-based condition monitoring of a gearbox
}

\author{
T Heyns ${ }^{\mathrm{a}, \mathrm{b}, *}$, PS Heyns ${ }^{\mathrm{c}}$, JP de Villiers ${ }^{\mathrm{a}, \mathrm{b}}$ \\ ${ }^{a}$ Department of Electronic and Computer Engineering, University of Pretoria \\ ${ }^{b}$ Defence Peace Safety and Security (DPSS) division of the Council for Scientific and Industrial Research (CSIR) of South Africa \\ ${ }^{c}$ Dynamic Systems Group, Department of Mechanical and Aeronautical Engineering, University of Pretoria
}

\begin{abstract}
This paper investigates how Gaussian mixture models (GMM) may be used to detect and trend fault induced vibration signal irregularities, such as those which might be indicative of the onset of gear damage. The negative log likelihood (NLL) of signal segments are computed and used as measure of the extent to which a signal segment deviates from a reference density distribution which represents the healthy gearbox. The NLL discrepancy signal is subsequently synchronous averaged so that an intuitive, yet sensitive and robust, representation may be obtained which offers insight into the nature and extent to which a gear is damaged. The methodology is applicable to non-linear, non-stationary machine response signals.
\end{abstract}

Keywords: Condition based maintenance, Negative log likelihood transform, Gaussian mixture model, Synchronous averaging

\section{Introduction}

Time synchronous averaging (TSA) has been proposed as early as 1975 [1], and is still recognised as an essential diagnostic procedure for condition monitoring of rotating equipment $[2,3,4]$. The TSA is computed as the ensemble average of the time domain signal over a number of shaft revolutions. Synchronous averaging requires an integer number of samples per shaft revolution. For this reason a preprocessing stage is often employed during which the signal is resampled at constant shaft angular intervals $[3,5]$. Since synchronous averaging generally requires signal resampling, this paper will omit the reference to the time domain and simply refer to synchronous averaging (SA), thereby implying that the procedure has been performed in the angular domain.

SA reduces noise and non-synchronous signal components, hence enhancing the signal characteristics of interest. Despite its general efficiency, SA suffers from certain limitations. Barszcz and Randall [5] perform a case study where a tooth crack in the planetary gear in a wind turbine cannot be detected by the SA approach. Further analysis by means of spectral kurtosis indicates that development of the failure could be detected even as early as 8 weeks in advance. Since the case study investigates a catastrophic failure from a real wind turbine the amount of available data is limited. Barszcz and Randall nevertheless conclude that SA is unlikely to detect the damage, even if sufficient data are available. This is because the detected peaks caused by the tooth crack have a very short duration, occur seldom and have varying amplitudes. It is concluded that the phase modulation induced

\footnotetext{
${ }^{*}$ Corresponding author

Email address: theoheyns@gmail.com (T Heyns)
} 
by the elastic deformation of the gear train components impede the SA process to such an extent that the high frequency pulses cannot be detected. Stander and Heyns [6] also investigate the amplitude and phase effects which are caused by the change in input torque to a gearbox. They propose a synchronous averaging methodology which compensates for the phase shifting in the measured structural response.

Residual signal analysis is a technique where non-fault related vibration components are removed from a signal so that only those signal components which are indicative of machine faults remain. A number of different residual signal methodologies have been investigated in literature. Wang and Wong [7] investigate how an autoregressive (AR) filter which is representative of the baseline vibration signal can be used to detect fault induced signal outliers in a novel signal. Heyns et al. [8] extend Wang and Wong's idea to better deal with time-varying operating conditions. This is done by implementing an assemble of weighted AR models, each of which represents the healthy vibration data, but for different operating conditions. When applied to a novel signal, the model weights are iteratively updated so as to better account for the instantaneous operating conditions, hence obtaining a residual signal which is more sensitive and robust under time-varying operating conditions. They also propose that the structure of the residual signal may be analysed to detect periodic events (outliers), as this may provide insight regarding the nature of the gear damage.

The AR filter based residual signal technique is conceptually related to a large group of methodologies which are concerned with outlier or novelty detection in data sets. Essentially these outlier or novelty detection methodologies are capable of modelling one class data (e.g. normal data) and then subsequently detecting samples (e.g. fault induced) which deviate from the baseline model. Some popular novelty or outlier detection methods include: rule based techniques, neural networks (including multilayer perceptrons, self organizing maps and auto-associative networks), support vector machines, Bayesian networks, nearest neighbour and distance techniques, as well as Gaussian Mixture Models (GMM) density based approaches [9]. Timusk et al. [10] conducts an interesting study where a number of novelty detection algorithms are used in conjunction with various signal preprocessing techniques to analyse data which were generated on an experimental gearbox test rig under time-varying operating conditions. The preprocessing and novelty detection techniques are used to extract robust features which are shown capable of discerning between a number of specified fault conditions. A related problem is that of speaker change detection, where the problem is to determine the time instances in an audio stream when a voice or speaker change. In recent years GMM-based change detection methodologies have served as the dominant approach for speaker change detection, primarily due to GMM's good ability to detect various acoustic changes [11].

This paper proposes a novel gear monitoring technique, which combines ideas from residual analysis, novelty detection and synchronous averaging. A Gaussian mixture model (GMM) is used to represent patterns in signal segments as observed for a healthy gearbox. The reference GMM density distribution is subsequently used to measure the extent to which short signal segments from a novel vibration measurement deviate from the healthy behaviour. The severity and the periodicity of the signal deviations are analysed for diagnostic information.

The proposed framework is illustrated on a simple analytical example which approximately represents vibration response signals from a gearbox which is subject to significant amplitude and phase modulation. The methodology is subsequently investigated on data which were generated on an experimental gear test rig. The test rig was subject to time-varying operating conditions. It is seen that GMM density distributions are potentially capable of detecting fault induced signal deviations in signals which are subject to fluctuating operating conditions. On the investigated data sets the negative log likelihood (NLL) synchronous averaging methodology outperforms conventional vibration signal synchronous averaging and spectral analysis.

\section{Methodology}

This paper proposes a methodology which combines concepts from residual analysis, novelty detection and synchronous averaging. The methodology comprise four key steps as illustrated in figure 1 . 


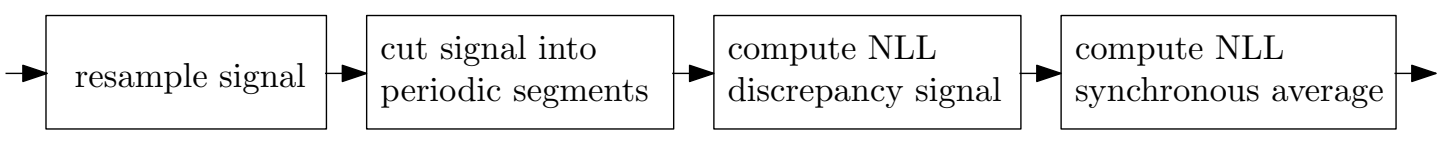

Figure 1: Diagram of the four key steps of the proposed NLL discrepancy signal synchronous averaging methodology.

1. The vibration signal is resampled at constant gear angular increments.

2. The resampled signal is segmented into block windows of fixed length. The signal segments are synchronous with specific shaft angular intervals. A window length may be chosen for instance to represent the (approximate) duration for which a gear-pinion tooth pair is in mesh.

3. The negative log likelihood (NLL) of each signal segment is computed. The NLL discrepancy signal is used as a measure of the extent to which individual signal segments deviate from the expected healthy behaviour.

4. All the NLL values which correspond to any particular gear (or pinion) angular interval are ensemble averaged. Gear angular intervals with small averaged NLL values correspond well to the expected behaviour, while large averaged NLL values indicate the possible presence of damage.

\section{Novelty detection with Gaussian Mixture Models}

The methodology implemented in this paper makes use of Gaussian mixture model (GMM) density distributions to compute the negative log likelihoods (NLL) of short signal segments. A linear combination of Gaussians can give rise to complex multivariate probability densities. Almost any continuous density function may be approximated to arbitrary precision by implementing sufficiently many Gaussian components with appropriately selected means, covariances and mixing coefficients $[12,13]$. GMM density estimates may thus be well suited to represent the possibly non-linear covariances or multiple density modes which may arise in signal sequences which have been generated under time-varying operating conditions. Individual mixture components may represent various, unobserved (latent) operating conditions. GMM models are fairly simple to implement, given that sufficient data are available for optimisation by means of expectation maximisation, and given that the length of the vector of random variables (length of the signal segment) does not give rise to very large dimensionalities. A probability density distributions may be used to generate a metric of similarity based on the natural logarithm of the likelihood of the observed sample [14] ${ }^{1}$. In this paper the negative natural logarithm of the likelihood (NLL) is used as a measure of discrepancy.

\section{Dividing the signal into segments}

The time domain vibration signal is resampled at constant increments of the shaft angular position. Signal resampling is implemented with piecewise-cubic interpolation and is based on a one pulse per shaft rotation tachometer reference signal. The resampled signal is subsequently cut into short signal segments by means of block windows.

The window length and the extent to which the windows overlap influence the resolution and sensitivity of the resulting NLL discrepancy signal. Longer window lengths offer more contextual information, however a long window length also increases the dimensionality of the reference density distribution. High dimensional density distributions as estimated with GMMs are more subject to local minima during optimisation and also to data overfitting, which may result in poor generalisation [12]. Long windows also result in increased smearing (reduced temporal resolution) of the NLL discrepancy signal.

\footnotetext{
${ }^{1}$ Please note that while probability and likelihood are related they are not interchangeable concepts. A likelihood value is obtained from a density distribution function when it is evaluated for a specific realisation of a continuous variable (or vector of variables). A probability is obtained by integrating a density distribution over a specified range of values which the random variable might take on. Probability is always limited to the interval [0-1], while likelihood is not.
} 
Consider that the signal segments may be generated in a manner similar to the sliding window approach implemented by a conventional auto-regressive (AR) filter in time-series analysis. Signal segments are created by moving the window one datum point at a time. The AR model does not differentiate between vibration signal segments which are generated at different shaft angular intervals. The output from the AR model is thus completely based on the contextual information which is contained in the previous number of signal datum points.

The NLL discrepancy signal implemented in this paper was initially investigated using a similar sliding window approach to that employed in conventional time-series analysis. While this approach was seen to work reasonably well, it was later realised that a more specific density distribution could be constructed if the signal segments are generated in such a manner that it inherently reflects knowledge of the structure of the signal.

In this paper it is assumed that only a single tachometer signal is available. If the tachometer is mounted on the gear shaft, then the gear angular position may be estimated in a consistent manner. Since the pinion generally rotates at a different frequency than the gear, it will not be known at the start of new measurements which pinion tooth is in mesh. If tachometers are mounted on both the pinion and the gear, or if it is only required to monitor the gear, then unique density models may be constructed for individual gear and pinion angular intervals.

In this paper the signal segments are generated such that they correspond to the periodicity of the gear meshing frequency. The number of windows per rotation of the gear is set equal to the number of teeth on the gear. The windows do not overlap, but immediately succeed each other. The gear mesh frequency often significantly contributes to (or dominates) the overall energy in the vibration signal. Signal segments with periodicities which correspond to the gear mesh frequency are generally much more similar, compared to signal segments selected at random. These segments may subsequently be used to obtain a density distribution which is subject to less variance, and which may subsequently result in more sensitive NLL discrepancy values. Figure 2 illustrates the implemented windowing methodology.

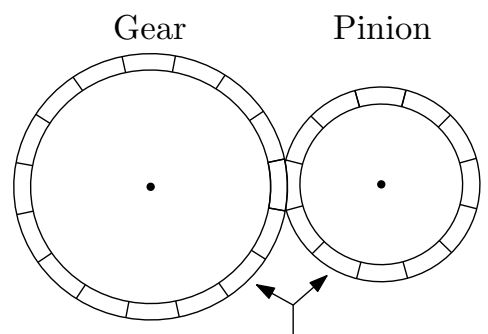

Fixed shaft angular intervals

Figure 2: Representation of the fixed window intervals which correspond to approximate pinion-gear meshing periods.

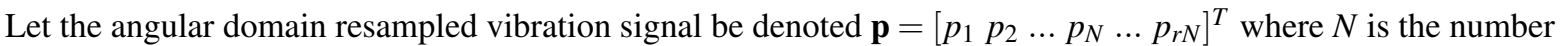
of resampled datum points per rotation of the shaft on which the tachometer is mounted The vibration signal is truncated so that all the data before the first and after the last tacho pulses are discarded. Let $r$ represent the integer number of complete shaft rotations during the measurement, so that $r N$ is the length of the resampled vibration signal. Let $T_{g}$ represent the number of teeth on the gear, and let $T_{p}$ represent the number of teeth on the pinion.

If the tachometer is mounted on the gear shaft, then the window length $L_{w}$ is obtained as the number of datum points per rotation of the gear, divided by the number of gear teeth $L_{w}=N / T_{g}$. Similarly if the tachometer is mounted on the pinion shaft then the window length is obtained as $L_{w}=N / T_{p}$. Let the first signal segment be represented by the column vector $\mathbf{s}_{1}=\left[\begin{array}{llll}p_{1} & p_{2} & \ldots & p_{L_{w}}\end{array}\right]^{T}$ and the second segment by $\mathbf{s}_{2}=\left[\begin{array}{llll}p_{L_{w}+1} & p_{L_{w}}+2 & \ldots & p_{2 L_{w}}\end{array}\right]^{T}$. This is repeated until $r N / L_{w}$ signal segments have been obtained. The signal segments are collected in the matrix $X_{s}$, where $X_{s}=\left[\begin{array}{lll}\mathbf{s}_{1} & \mathbf{s}_{2} \ldots \mathbf{s}_{r N / L_{w}}\end{array}\right]$. Each column in $X_{s}$ represents a pinion-gear meshing period. 
A measure of discrepancy (NLL value) will be computed for each column in $X_{s}$. The resulting NLL discrepancy signal will have a much lower sampling rate than the original vibration signal. The lower sampling rate reduces the computational burden of the algorithm, without discarding diagnostic information. The NLL sampling frequency corresponds to one value for each pinion-gear mesh. The NLL discrepancy signal may thus be synchronous averaged with respect to either the gear or the pinion without the need for additional resampling.

\section{Density estimation with Gaussian mixture models}

A Gaussian mixture model (GMM) is optimised to represent the density distribution which corresponds to signal segments from the healthy gearbox. Alternative non-parametric methods, such as density estimation with Parzen windows, may also be considered. However, non-parametric approaches tend to be both memory and computationally intensive, while also being more prone to data overfitting, especially for high dimensional density distributions [13].

A GMM comprises a weighted super positioning of individual multivariate Gaussian components [12]. The complexity of a GMM may be controlled by limiting the number of mixture components, or by constraining the covariance matrices. When sufficient training data are available GMMs may be used to estimate very complex density distributions $[12,13]$. Mixture models are for instance well suited to represent data which might be generated by different classes. The classes may be unknown and thus treated as latent variables [13]. A density distribution may thus represent vibration signal segments which have been generated under different operating conditions, but of which the operating conditions is unobserved (latent). A mixture model with $K$ components is denoted as [12]:

$$
p(\mathbf{g})=\sum_{k=1}^{K} \pi_{k} N\left(\mathbf{g} \mid \mu_{k}, \Sigma_{k}\right),
$$

where the mixing coefficients $\pi_{k}$ must sum to $1, \sum_{k=1}^{K} \pi_{k}=1$. Given that $N\left(\mathbf{g} \mid \mu_{k}, \Sigma_{k}\right) \geq 0$ a sufficient condition that $p(\mathbf{g}) \geq 0$ is that $\pi_{k} \geq 0$ for all $k$. If each of the individual components are valid, and the aforementioned constraints are satisfied, then the resulting mixture model will also be a valid density function which integrates to $1[12]$.

The model parameters are optimised in Matlab using expectation maximisation (EM) so as to maximise the likelihood of the observed data. A good reference on the topic of expectation maximisation is provided by Bishop [12]. The EM algorithm is initialised by centring the GMM components on randomly selected samples from the training set. The EM algorithm is not guaranteed to find the global optimum, so that it is expected that the performance from different optimisation runs will differ slightly. The EM optimisation step is thus repeated a number of times, and the solution which offers the best results is subsequently selected.

\section{Conditional density distributions}

Occasionally the operating conditions (or at least covariates which partially describe the operating conditions) may be measured. It may then be possible to use the knowledge of the operating conditions to refine the computed NLL values. This may be done by conditioning the outcome of the observed signal segment on the measured operating conditions. This should render the computed NLL values more robust to time-varying operating conditions, and subsequently more sensitive to fault induced signal outliers.

Consider for example that the shaft angular speed (as possibly estimated from the tachometer) may occasionally be proportional to the applied torque on a gearbox. The angular speed may subsequently co-vary (possibly in a non-linear manner) with the amplitude or phase modulation of a signal segment. 
As previously defined the column vector $\mathbf{s}_{i}$ is used to represent a single signal segment. Let $\mathbf{c}_{i}$ represent the column vector which contains variables which are representative of the observed operating conditions which corresponds to the period during which the segment $\mathbf{s}_{i}$ is measured. The operating conditions vector $\mathbf{c}_{i}$ may for example contain one entry which represents the mean angular velocity and a different one which describes the applied load.

The likelihood of a signal segment $\mathbf{s}_{i}$ as conditioned on the vector describing the associated operating conditions $\mathbf{c}_{i}$, is equal to the joint likelihood of both $\mathbf{s}_{i}$ and $\mathbf{c}_{i}$, divided by the likelihood of $\mathbf{c}_{i}$, so that $P\left(\mathbf{s}_{i} \mid \mathbf{c}_{i}\right)=P\left(\mathbf{s}_{i}, \mathbf{c}_{i}\right) / P\left(\mathbf{c}_{i}\right)$ [12].

A GMM may first be used to estimate a joint density distribution for both the signal segments and the operating conditions as contained in the matrix $X_{s c}$,

$$
\mathbf{X}_{\mathbf{s c}}=\left[\begin{array}{cccc}
\mathbf{s}_{1} & \mathbf{s}_{2} & \ldots & \mathbf{s}_{r N / L_{w}} \\
\mathbf{c}_{1} & \mathbf{c}_{2} & \ldots & \mathbf{c}_{r N / L_{w}}
\end{array}\right]
$$

A separate GMM may then be estimated for the operating conditions contained in matrix $X_{c}=\left[\mathbf{c}_{1} \mathbf{c}_{2} \ldots \mathbf{c}_{r N / L_{w}}\right]^{2}$. The conditional NLL value, $y$, for any signal segment may then be computed, $y_{i}=-\ln \left[P\left(\mathbf{s}_{i}, \mathbf{c}_{i}\right) / P\left(\mathbf{c}_{i}\right)\right]$.

\section{Model selection}

The more components a mixture model has the more expressive (more degrees of freedom) it becomes, theoretically allowing for a better representation of the density distribution of the data. Complex models are however also more prone to data overfitting, which may result in poor generalisation [12]. A model which does not generalise well may detect many false signal outliers, hence resulting in decreased performance.

There are a number of methods which may be considered for selecting the optimal number of mixture components. Some popular methodologies include: the Akaike information criterion, Bayes' information criterion, and Bayesian model selection. Since data of the gearbox in its healthy condition may generally be assumed to be fairly abundant, and due to its ease of implementation, this paper resorts to model selection based on likelihood cross-validation as discussed by Smyth [15].

Cross-validation is a popular and simple method for measuring the ability of a model to generalise well [12] and Smyth [15] concludes that it is also well suited to selecting the number of components for a GMM, particularly when data and computational resources are relatively plentiful.

In essence the data which represent the healthy gearbox are divided into two sets. The first set is used to optimise the GMM by means of expectation maximisation. The second set is then used to measure the ability of the trained GMM to generalize well on novel data. This approach is used to investigate the appropriateness of different numbers of mixture components.

\section{Synchronous averaging}

In this paper a special case of the synchronous average is implemented. The NLL discrepancy signal $\mathbf{y}$ has a sampling frequency (samples per rotation of the gear) which is equal to the number of teeth on the gear. Similarly

\footnotetext{
${ }^{2}$ The Gaussian distribution has the favourable characteristic that it is particularly easy to marginalise over variables. This renders it possible to avoid the necessity to train a second GMM model. The density distribution which only represents the operating conditions can be obtained from the full distribution which explain the joint signal segments and operating conditions distribution. This is done by simply discarding appropriate indices from the mean vector and covariance functions. The reader is referred to the book by Bishop [12] for additional reference to marginalisation in Gaussian distributions
} 
is the number of NLL datum points per rotation of the pinion equal to the number of teeth on the pinion. For this reason no further resampling of the NLL discrepancy signal will be required. The number of full shaft rotations during the measurement is represented by $r$. The gear synchronous average $\overline{\mathbf{y}}^{g}$ is computed as:

$$
\bar{y}_{i}^{g}=\frac{1}{r} \sum_{n=1}^{r^{g}} y_{n \times T_{g}}, \text { for } i=1 \ldots T_{g}
$$

where $\bar{y}_{i}^{g}$ represents the synchronous average associated with the $i$ th tooth on the gear and $r^{g}$ is the number of complete revolutions completed by the gear during the measurement. Similarly the pinion synchronous average $\overline{\mathbf{y}}^{p}$ is computed as:

$$
\bar{y}_{i}^{p}=\frac{1}{r} \sum_{n=1}^{r^{p}} y_{n \times T_{p}}, \text { for } i=1 \ldots T_{p}
$$

where $\bar{y}_{i}^{p}$ represents the synchronous average associated with the $i$ th tooth on the pinion and $r^{p}$ is the number of complete revolutions completed by the pinion during the measurement.

\section{Analytic investigation}

A simple analytical example is used to illustrate the underlying approach followed by the NLL discrepancy analysis technique. The analytic example is based on the gear model developed by McFadden [16], and which is also implemented by Combet and Gelman [17]. A vibration signal for a one stage gearbox is approximated by the following model:

$$
p(t)=\sum_{m=0}^{M} P_{h}\left(1+a_{h}(t)\right) \cos \left(2 \pi h f_{m} t+\theta_{h}(t)+\theta_{h}^{0}\right)+n(t)
$$

where $P_{h}$ is the amplitude of the $h$ th mesh harmonic, $f_{m}$ is the average mesh frequency for the stage considered, $a_{h}(t)$ and $\theta_{h}(t)$ are the amplitude and phase modulation functions of mesh harmonic $h$ and $\theta_{h}^{0}$ is the initial phase of harmonic $h$. The additive background noise $n(t)$ is assumed to be independently sampled from a zero mean normal distribution with a standard deviation of $\sigma$.

The mesh frequency $f_{m}$ is proportional to the rotational speeds $f_{p}$ and $f_{g}$ of the pinion and gear, through the number of teeth $T_{p}$ and $T_{g}$ on the pinion and the gear.

$$
f_{m}=T_{p} f_{p}=T_{g} f_{g}
$$

The healthy vibration signal is approximated by 3 harmonic components, $M=3$. The amplitudes $P_{h}$ of all three components are simply set to 1 . The signal is generated with $10 \mathrm{k}$ samples per revolution of the pinion.

The number of teeth on the pinion and the gear are respectively selected as $T_{p}=20$, and $T_{g}=43$. A measurement of $3 \mathrm{~s}$ long is generated. During this time the gearbox is subject to a time-varying load. It is assumed that the pinion shaft speed is directly proportional to the applied torque. During the first $0.6 \mathrm{~s}$ of the signal the gearbox is 
Table 1: Instantaneous pinion rotational frequency

time interval pinion rotational frequency

\begin{tabular}{ll}
\hline first $0.4 \mathrm{~s}$ & $f_{p}(t)=5 \times[1+0.3 \sin (1.3 \pi t)]$ \\
last $2.6 \mathrm{~s}$ & $f_{p}(t)=5 \times[1+0.5 \sin (5 \times 1.3 \pi t)]$
\end{tabular}

Table 2: Instantaneous amplitude modulation

\begin{tabular}{ll} 
time interval & amplitude modulation function \\
\hline first $0.4 \mathrm{~s}$ & $a_{h}(t)=0.3 \sin (1.3 \pi t)$ \\
last $0.6 \mathrm{~s}$ & $a_{h}(t)=0.5 \sin (5 \times 1.3 \pi t)$
\end{tabular}

subject to a rapid and significant sinusoidal load fluctuation, during the last $2.4 \mathrm{~s}$ the gearbox is subject to a smaller, more gradual sinusoidal load fluctuation. Due to the presence of the time-varying operating conditions, the signal experiences frequency, amplitude and phase modulation.

The pinion has a mean rotational frequency of $E\left(f_{p}\right)=5 \mathrm{~Hz}$. Its instantaneous frequency $f_{p}(t)$ (due to the time-varying operating conditions) is indicated in table 1:

The amplitude and phase modulation are set proportional to the time-varying pinion shaft speed. The amplitude modulation $a_{h}$ is similar for each of the tooth meshing harmonic components $h=1,2,3$ as indicated in table 2 .

The phase modulation $\theta_{h}$ is also proportional to the instantaneous pinion frequency for each of the harmonics $h=1,2,3$. However the phase modulation must also account for the wavelength of the harmonic. Short wavelengths must be phase modulated proportionally more than long wavelengths in order to ensure that the different phase components are not distorted and do not move relative to one another. The resulting phase modulation is presented in table 3

The initial phase $\theta_{h}^{0}$ of each of the harmonics is simply set to 0 .

A single fault is simulated on the tenth pinion tooth. If the gear has a local defect such as a tooth root crack, then changes will occur in the vibration when the affected teeth are in mesh. McFadden [16] represents a localised gear crack by means of amplitude and phase modulation functions which are periodic with the gear rotational frequency. This assumption may result in a fairly deterministic fault component. The case study performed by Barszcz and Randall [5] however indicates that localised gear tooth cracks may give rise to high frequency (impulsive like) signal components, of which the amplitude may have a large variance. The experimental study performed in this paper indicates similar fault behaviour.

The pinion tooth damage is simulated in this paper by assuming that the tooth root crack gives rise to a relatively high frequency signal component. This is simulated by appending a tenth order harmonic to the signal, which obeys the same operating condition related amplitude and phase modulation rules as the first three (healthy gear)harmonic components. It is assumed that the high frequency component is only exited when the faulty tooth is in mesh, and then quickly dies out. This is done by amplitude modulating the 10th harmonic by an asymmetric saw-tooth waveform. The saw-tooth waveform is created in Matlab using the Tripuls function. The saw-tooth has a repetition frequency which corresponds to the rotational frequency of the pinion. The saw-tooth width is equal to the period that the 11 th tooth is in mesh. In other words the 10 th harmonic has zero energy at all positions, except for the approximate duration that the faulty tooth is in mesh. It will be seen that the high frequency 10 th harmonic, which is subject to significant load induced amplitude and phase modulation will behave sufficiently stochastic so that conventional synchronous averaging will fail to detect it. 
Table 3: Instantaneous phase modulation

\begin{tabular}{ll} 
time interval & phase modulation function \\
\hline first $0.4 \mathrm{~s}$ & $\theta_{h}=0.2 h \times 0.5 \sin (5 \times 1.3 \pi t)$
\end{tabular}

last $2.4 \mathrm{~s} \quad \theta_{h}=0.2 h \times 0.3 \sin (1.3 \pi t)$
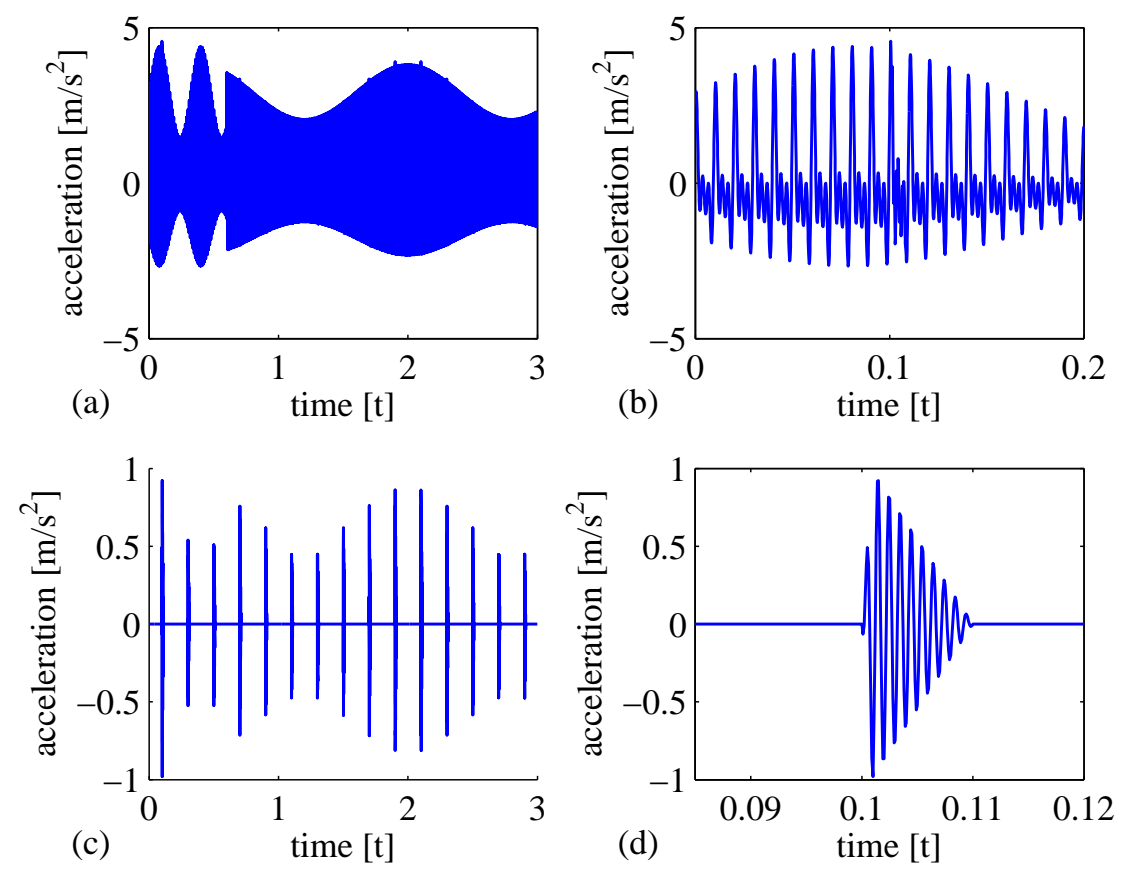

Figure 3: (a) Simulated damaged vibration waveform subject to amplitude and phase modulation, (b) enlarged view of the simulated vibration signal, (c) the saw tooth amplitude modulated 10th order harmonic component which represents the transient fault induced signal component, (d) an enlarged view of one of the fault induced signal transients indicates the saw-tooth nature of the envelope of the component.

Figures 3 (a)-(d) investigate the nature of the simulated vibration waveform. Figure 3 (a) illustrates the noise-free, damaged vibration waveform, which is sampled at $10 \mathrm{k}$ datum points per revolution of the pinion. This results in 500 data points per signal segment. In Figure 3 (b) the noise free vibration signal is magnified, such that a single complete revolution of the pinion is visible. The fault induced signal distortion is seen at $0.1 \mathrm{~s}$. Figure 3 (c) shows the 10th harmonic (fault induced) signal component. The 10th harmonic is subject to the same load induced amplitude modulation experienced by the other 'healthy' harmonics, but is also transient due to its amplitude multiplication with the saw-tooth waveform. Figure 3 (d) presents an enlarged view of a single fault induced signal transient. 

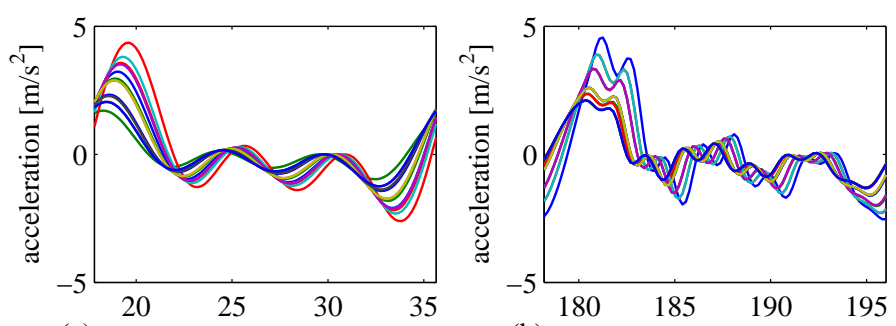

(a) pinion angular pos $[\mathrm{deg}]$

(b) pinion angular pos $[\mathrm{deg}]$

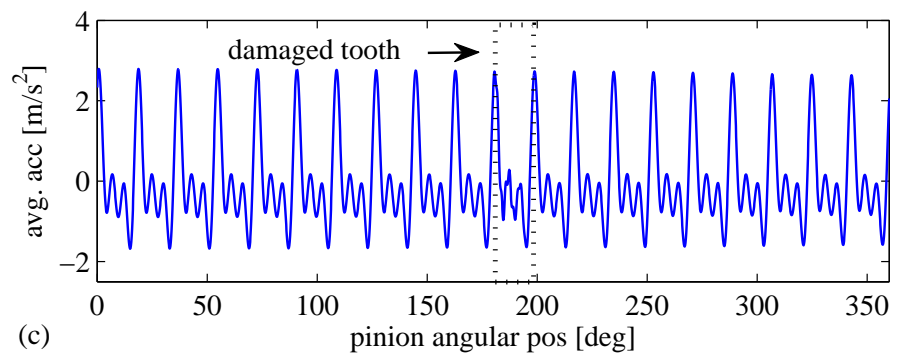

Figure 4: (a) Overlay of multiple signal segments from a healthy pinion tooth, indicating both amplitude and phase modulation, (b) overlay of multiple signal segments from the damaged pinion tooth, indicating how the higher frequency components significantly smears out (c) the resulting (noise free) synchronous average with respect to the pinion. The damage induced signal distortion on the 11th tooth (angular position 180-198 degrees) is almost invisible.

Figure 4 (a) illustrates the nature and extent of the amplitude and phase modulation present in signal segments which corresponds to a healthy tooth. The signal segments are presented without any additive noise. Each signal segment is 500 datum points long and corresponds to a tooth meshing period. Figure 4 (b) investigates the effect of the amplitude and phase modulation on signal segments which corresponds to the 11th (damaged) pinion tooth. Figure 4 (c) indicates the conventional vibration signal synchronous average. The high frequency component is smeared out due to the load induced phase modulation. This renders the damage on the 11th tooth near invisible in the conventional synchronous average. The synchronous average is computed on a noise free signal, so that the small amount of damage on the 11th tooth may be observed. As soon as signal noise is added it becomes impossible to identify the damaged gear tooth. 

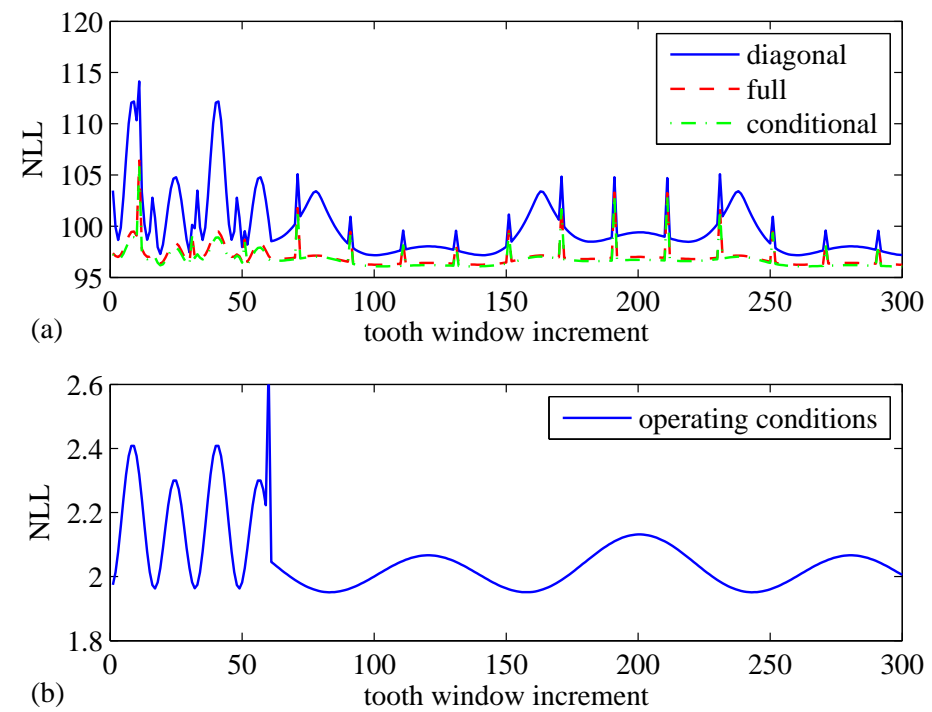

Figure 5: (a) Illustrates the NLL discrepancy signals as computed for the vibration signal, the continuous line represents NLL values $-\ln [P(\mathbf{s})]$ computed with a GMM which is limited to 2 components with diagonal covariance matrices, the dashed line represents a GMM with 2 components which have unconstrained covariance matrices, and the dash-dotted line (which closely corresponds to the dashed line) represents NLL values as conditioned on the pinion shaft angular velocity $-\ln \left[P\left(\mathbf{s}_{\mathbf{i}}, \mathbf{c}_{\mathbf{i}}\right) / P\left(\mathbf{c}_{i}\right)\right]$. (b) The NLL of the operating conditions $\ln \left[P\left(\mathbf{c}_{i}\right)\right]$.

A GMM density distribution is used to represent the signal segments which correspond to the fault free data. The vibration signal is sampled at $10 \mathrm{k}$ datum points per rotation of the pinion. Since there are 20 teeth on the pinion the signal segments have a length of 500 data points. The window length of 500 data points results in a GMM with many free parameters. In general it might be beneficial to consider down sampling the vibration signal so that a smaller GMM may be implemented. Alternatively it may also be considered to extract and model a subset of features (e.g. through principal component, or wavelet analysis). Lastly may it also be considered to constrain the covariance matrices, for instance by implementing diagonal covariance matrices.

Three approaches are investigated towards implementing the proposed strategy. The GMMs are implemented on vibration signals which were subject to random noise. A noise standard deviation of $\sigma=0.25$ is implemented. This noise level renders it completely impossible to identify the faulty gear tooth by means of conventional synchronous averaging.

The first approach is implemented by using a GMM with constrained covariance matrices. A diagonal covariance matrix is not capable of modelling the covariance between different datum points, but is also less prone to overfit data. GMMs with different numbers of mixture components are investigated. It is found that a single component with a constrained diagonal covariance is especially poor at discerning between time-varying operating conditions and fault induced signal outliers. Performance is significantly improved by implementing 2 components, but it is found that at least 10 components are required to obtain results which are comparable with a 1 component full rank covariance GMM model. The performance of a 2 components GMM with diagonal covariance matrices are indicated in figure 5 (a) with the solid (blue) line. The NLL values are seen to be sensitive to the operating conditions.

The second approach implements a GMM with full rank covariance matrices. Full rank covariance matrices render it possible to model the interdependencies between different datum points in a signal segment. Even a single full rank covariance component performs quite well in this simple analytical example. The dashed line in figure 5 (a) indicates the NLL values as obtained with a 2 component GMM with full rank covariance matrices. 
The third and final approach investigates the use of conditional NLL values. To compute any single conditional negative $\log$ likelihood value $-\ln \left[P\left(\mathbf{s}_{i}, \mathbf{c}_{i}\right) / P\left(\mathbf{c}_{i}\right)\right]$ it is required to estimate two GMM density distributions, one which represents the joint density distribution $P\left(\mathbf{s}_{i}, \mathbf{c}_{i}\right)$ for the signal segments $\mathbf{s}_{i}$, for $i=1 . . r N$ and the operating condition variable(s) $\mathbf{c}_{i}$, for $i=1 . . r N$, and a second density distribution $P\left(\mathbf{c}_{i}\right)$ which represents the operating conditions. Only a single operating condition variable is investigated, so that the vector $\mathbf{c}_{i}$ reduces to a variable $c_{i}$. The pinion angular velocity is used as operating condition variable $c$, since it is known to be indicative of the amplitude and phase modulation. Both density distributions are estimated with 2 component GMM models with full rank covariance matrices.

Figure 5 (b) indicates the computed NLL for the operating conditions. It is seen that the NLL values for the operating conditions which correspond to the first $0.4 \mathrm{~s}$ generally have large values. This shows that the first $0.4 \mathrm{~s}$ of operating conditions are not well representative of the typical operating conditions. A large spike is observed at the end of the $0.4 \mathrm{~s}$. This represents the transition from one operating condition to the other. The large spike indicates that this transitional operating condition is very uncommon.

The NLL discrepancy signal obtained for the GMM with the diagonally constrained covariance matrices is represented by the continuous line in figure 5 (a). The limited covariance matrix used by the GMM results in a NLL discrepancy signal which is sensitive to the operating conditions and subsequently not well suited for detecting the fault induced signal component.

The NLL values obtained for the full rank covariance GMM model is represented by the dashed line, and the dash-dotted line illustrates the conditional NLL values. The NLL discrepancy signal obtained for both the full rank covariance GMM model and the conditional NLL discrepancy signal are very similar. This indicates that the joint density distribution did not manage well to model the non-linear correlation between the signal segments and the pinion rotational frequency. In this scenario conditioning the NLL on the operating condition did not serve to render the NLL more robust to the time-varying operating conditions. However the NLL values obtained for the operating conditions (figure 5 (b)) may still be beneficial. Those sections of the vibration signal which were generated under operating conditions which are not well representative of the typical operating conditions may be discarded. For instance, figure 5 (b) may be used to determine that the first 0.4 seconds of signal should be discarded and not used to compute the NLL synchronous average. 

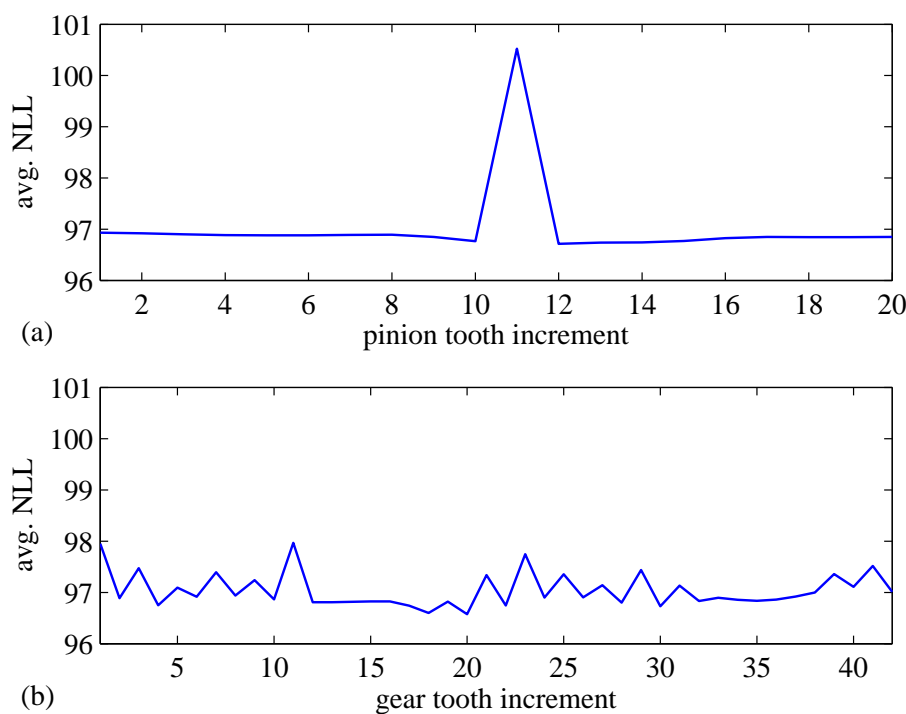

Figure 6: (a) The synchronous averages of the signal segment NLL values which correspond to the 20 teeth on the pinion, and (b) the synchronous averages which correspond to the 43 gear teeth.

Figures 6 (a) and (b) respectively indicate the NLL synchronous averages with respect to the pinion, and the gear. The NLL signals are generated with the unconditional full rank covariance GMM with 2 components. These synchronous averages are computed by using the whole (noisy) NLL signal, which includes the first $0.4 \mathrm{~s}$ during which the gear was subject to significant fluctuating operating conditions. The pinion completed 14 whole rotations, while the gear only completed 6 . A single averaged datum point corresponds to each gear mesh period. This renders it possible to synchronous average the NLL values with respect to both the pinion and the gear without the need to resample the NLL signal. A large average NLL value corresponds to the 11th pinion tooth, clearly indicating the presence of the fault induced signal irregularity.

In this simple analytical example, the NLL synchronous average was able to clearly detect tooth damage, which could not be detected by conventional synchronous averaging.

\section{Experimental setup}

The proposed NLL synchronous averaging methodology is subsequently investigated on experimental data which were recorded in the Sasol Laboratory for Structural Mechanics at the University of Pretoria [18]. 


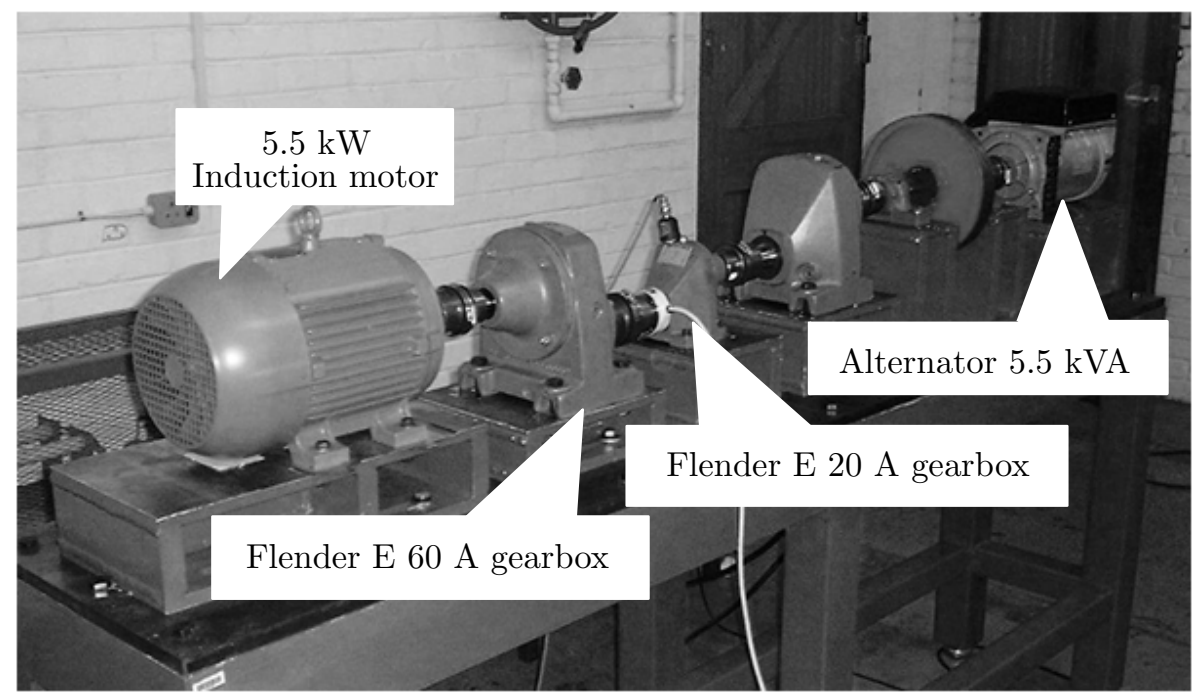

Figure 7: The experimental setup of the gear test rig.

Gear damage was investigated on a single stage Flender E20A gearbox (rated load of $20 \mathrm{Nm}$ ) with helical gears and a step up ratio of 1:1.96. A mounted test bed was designed to conduct accelerated gear life tests on the gearbox under time-varying load conditions. As such the monitored gearbox was mounted between two Flender E60A gearboxes, the first of which served to step the torque up and the second to step it down. The E60A gearbox pair increased the torque over the E20A test gearbox by a ratio of 1:4.72. The fundamental system frequencies are presented in table 4 .

Table 4: Fundamental frequencies

\begin{tabular}{|l|l|l|l|l|l|}
\hline Gearbox & Role & Shaft input & Teeth ratio & Shaft output & Mean Meshing Frequency \\
\hline Flender E60A & Speed step down & $24.5 \mathrm{~Hz}$ & $18 / 85$ & $5.19 \mathrm{~Hz}$ & $441 \mathrm{~Hz}$ \\
\hline Flender E20A & Test gearbox, speed step up & $5.19 \mathrm{~Hz}$ & $43 / 22$ & $10.13 \mathrm{~Hz}$ & $223 \mathrm{~Hz}$ \\
\hline Flender E60A & Speed step up & $10.13 \mathrm{~Hz}$ & $85 / 18$ & $47.88 \mathrm{~Hz}$ & $862 \mathrm{~Hz}$ \\
\hline
\end{tabular}

The step down gearbox was driven by a $5.5 \mathrm{~kW}$ three phase four pole WEG squirrel cage electric motor. The step up gearbox was connected to a flywheel which in turn was coupled with a $5.5 \mathrm{kVA}$ Mecc Alte Spa three phase alternator to apply the load.

The instantaneous gear and pinion angular positions and velocities were estimated from a synchronising pulse which was measured by means of a proximity switch on the key of the E20A gear shaft. The gear casing acceleration response was measured in the vertical direction by means of a $10 \mathrm{~V} / \mathrm{g}$ PCB integrated circuit piezoelectric industrial accelerometer. The measurements were taken with a Siglab model 20-42 signal analyser at a sampling frequency of $51.2 \mathrm{kHz}$.

A number of time-varying loading conditions were investigated. The load conditions are summarized in Table 5. 
Table 5: Load case specifications

\begin{tabular}{|c|c|c|c|c|}
\hline Load case & Load function & Frequency & Minimum load & Maximum load \\
\hline 1 & Sine & $0.5 \mathrm{~Hz}$ & $7.4 \mathrm{Nm}$ & $14.7 \mathrm{Nm}$ \\
\hline 2 & Sine & $1 \mathrm{~Hz}$ & $7.4 \mathrm{Nm}$ & $14.7 \mathrm{Nm}$ \\
\hline 3 & Square & $0.5 \mathrm{~Hz}$ & $7.4 \mathrm{Nm}$ & $14.7 \mathrm{Nm}$ \\
\hline 4 & Square & $1 \mathrm{~Hz}$ & $7.4 \mathrm{Nm}$ & $14.7 \mathrm{Nm}$ \\
\hline 5 & Chrip & $0.1-2 \mathrm{~Hz}$ & $7.4 \mathrm{Nm}$ & $14.7 \mathrm{Nm}$ \\
\hline 6 & Random & $0.1-2 \mathrm{~Hz}$ & $7.4 \mathrm{Nm}$ & $14.7 \mathrm{Nm}$ \\
\hline
\end{tabular}

Four measurements for each load condition were recorded. The first measurement represented the healthy gearbox. Flank wear was subsequently induced on one of the gear teeth by progressively removing more of the gear tooth face. Each measurement was recorded for 30s.

Table 6: Seeded gear damage condition

\begin{tabular}{|c|c|}
\hline Measurement & Fault severity \\
\hline 0 & Good condition \\
\hline 1 & $100 \mu m$ tooth face removal \\
\hline 2 & $200 \mu m$ tooth face removal \\
\hline 3 & $300 \mu m$ tooth face removal \\
\hline
\end{tabular}

The subsequent sections investigate the vibration signal, the vibration based synchronous average, the spectrum analysis, the NLL discrepancy transform and the NLL synchronous average. The magnitude of the results vary slightly depending on the load scenario, however the nature of the results remain fairly consistent. For this reason one load scenario, namely the $0.5 \mathrm{~Hz}$ sinusoidal load scenario will consistently be used to visualise the application of the techniques.

\section{Time domain waveform}

The time domain waveform for load scenario 2, with the $0.5 \mathrm{~Hz}$ sinusoidal component, is illustrated in figures 8 (a)-(d), where (a) represents the healthy waveform, and (b)-(d) represent progressive damage. The sinusoidal component of the load induces periodic amplitude and phase modulation in the signal. As the damage increase the presence of signal impulses becomes apparent. In general the kurtosis values for the signals increase for the 2nd and especially the 3rd damage condition. The first damage condition can usually not be detected based on the kurtosis of the signal. The magnitude of the impulses, as observed for any one damage condition and any one loading scenario, tends to vary significantly. 

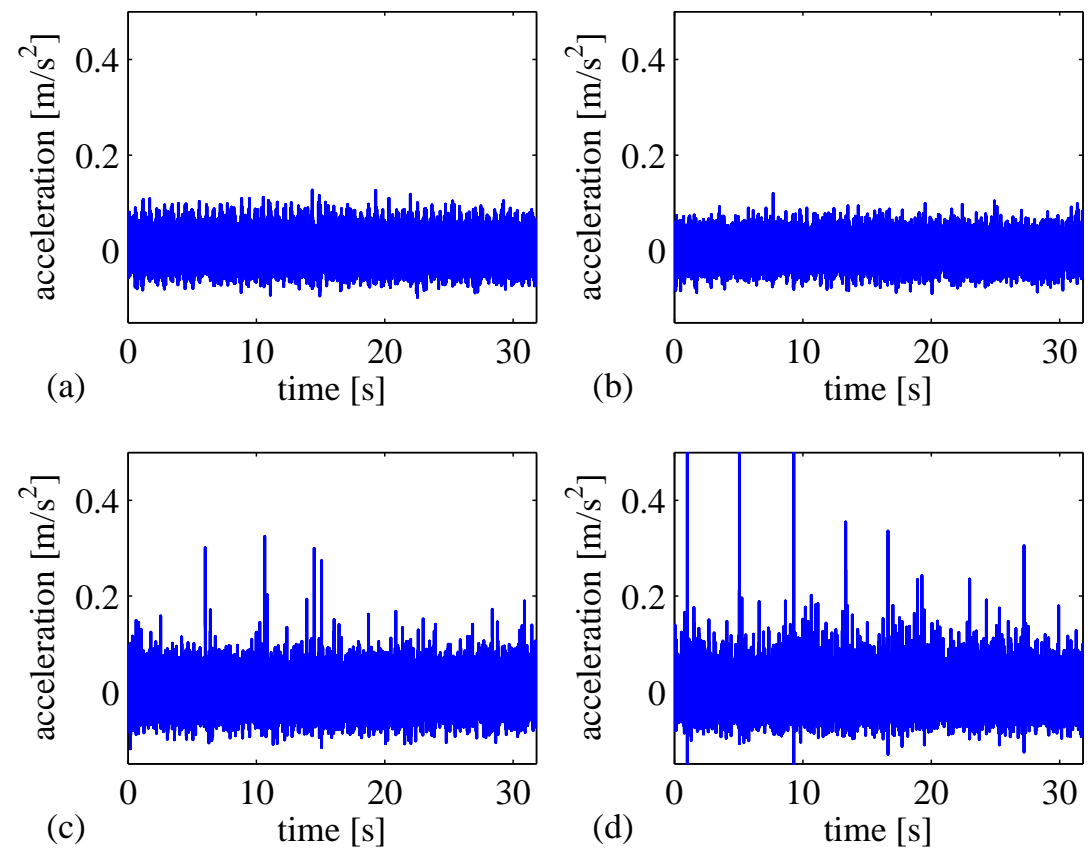

Figure 8: Vibration signal generated under $0.5 \mathrm{~Hz}$ sinusoidal loading condition, for (a) the healthy gear, and (b)-(d) progressive stages of damage.

\section{Power spectral density (PSD)}

The order domain power spectral densities (PSD) of the vibration signals are investigated. Towards this goal the time domain signals are resampled to the order domain by means of piecewise cubic interpolation of the once per gear revolution impulses as measured with the proximity probe.

Before resampling is performed the signal is further low-pass filtered to avoid aliasing. The time-domain signal which has a sampling frequency of $51.2 \mathrm{kHz}$ is resampled at 8600 datum points per revolution of the gear. The gear has an average rotational frequency of $5.19 \mathrm{~Hz}$, so that the angular domain resampling of 8600 datum points approximately corresponds to a time domain sampling rate of $44.6 \mathrm{kHz}$. To avoid aliasing during the resampling period an eighth-order lowpass Chebyshev Type I filter is used with a cut-off frequency of $35 \mathrm{kHz}$.

The natural logarithms for the order domain PSDs as computed for the $0.5 \mathrm{~Hz}$ sinusoidal time-varying load are presented in figures 9 (a)-(d), where (a) represents the gear in a good condition, and (b)-(d) represent progressive stages of damage. The first order corresponds to the gear rotational frequency of $5.19 \mathrm{~Hz}$.

The PSD for each signal is computed as follows. The signal (of approximate length $1.4 \times 10^{6}$ ) is divided into 3 , partially overlapping sections of $2^{19}$ datum points each. Each of the sections are Hanning windowed. Their FFTs (Fast Fourier Transforms) are then computed before the results are averaged. The FFTs are subsequently multiplied by their complex conjugates to obtain the PSD values. To compensate for the Hanning window the signal amplitude is corrected by factor 8/3. The PSD window length is selected to offer a compromise between spectral resolution, and the noise level of the PSD. 

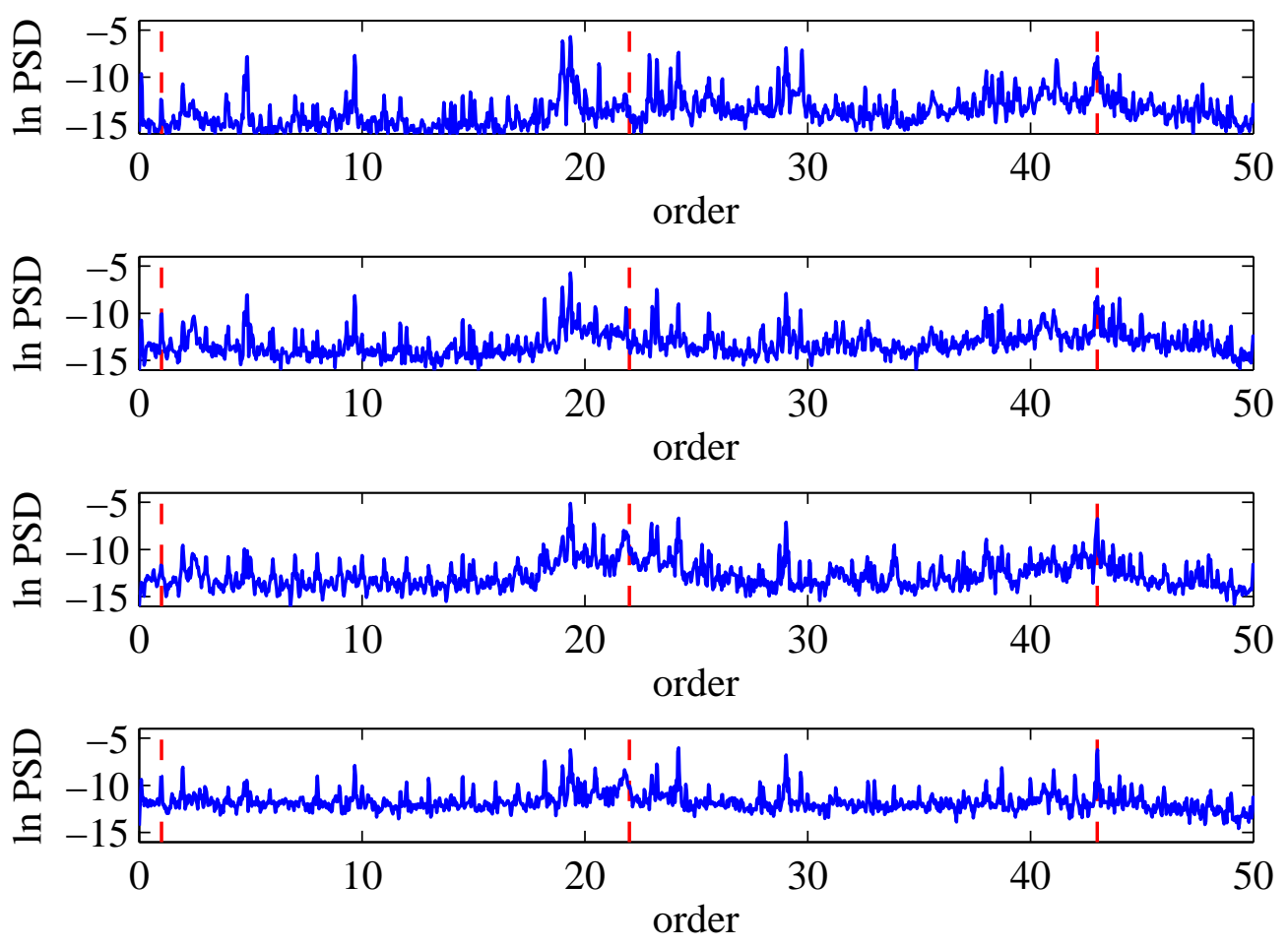

Figure 9: The order domain PSDs of the vibration signals as measured under the $0.5 \mathrm{~Hz}$ loading condition for (a) no damage, and (b) - (d) progressive stages of damage.

Significant energy is observed at approximately the 2 nd order $(10.14 \mathrm{~Hz})$ and the 4.8 th order $(24.5 \mathrm{~Hz})$, which respectively correspond to the drive shaft frequencies for the E60A step up and step down gearboxes. A prominent peak is observed at the 19.3 th order $(100 \mathrm{~Hz})$. This peak corresponds to twice the $50 \mathrm{~Hz} \mathrm{AC}$ line frequency. Generally the $100 \mathrm{~Hz}$ component tends to be more prominent in motors and alternators than the AC frequency component. Side bands due to amplitude modulation are observed around this $100 \mathrm{~Hz}$ frequency, this is especially noticeable in figures 9 (a) and (b). Large signal energy components are also identified at the following orders; the 28.9th $(150 \mathrm{~Hz})$, the 33.7th $(175 \mathrm{~Hz})$, the 38.5th $(200 \mathrm{~Hz})$, and the 43th $(223 \mathrm{~Hz})$. The $150 \mathrm{~Hz}$ and 200 $\mathrm{Hz}$ components may be harmonics which correspond to the excitation induced by the AC line current. The 175 $\mathrm{Hz}$ component might be due to amplitude modulation between the $200 \mathrm{~Hz}$ component and the $24.5 \mathrm{~Hz}$ E60A drive shaft frequency component. The 43th order component corresponds to the gear meshing frequency of the Flender E20A gearbox of interest.

While it is not indicated on the current axis, energy components which correspond to the E60A step down gearbox meshing frequency of $441 \mathrm{~Hz}$ and the E60A step up gearbox meshing frequency of $862 \mathrm{~Hz}$ may also be observed on the extended PSD.

The frequency components which are of interest with regard to the condition of the gear in the Flender F20A gearbox are magnified for better inspection in figures 10 (a)-(h). 

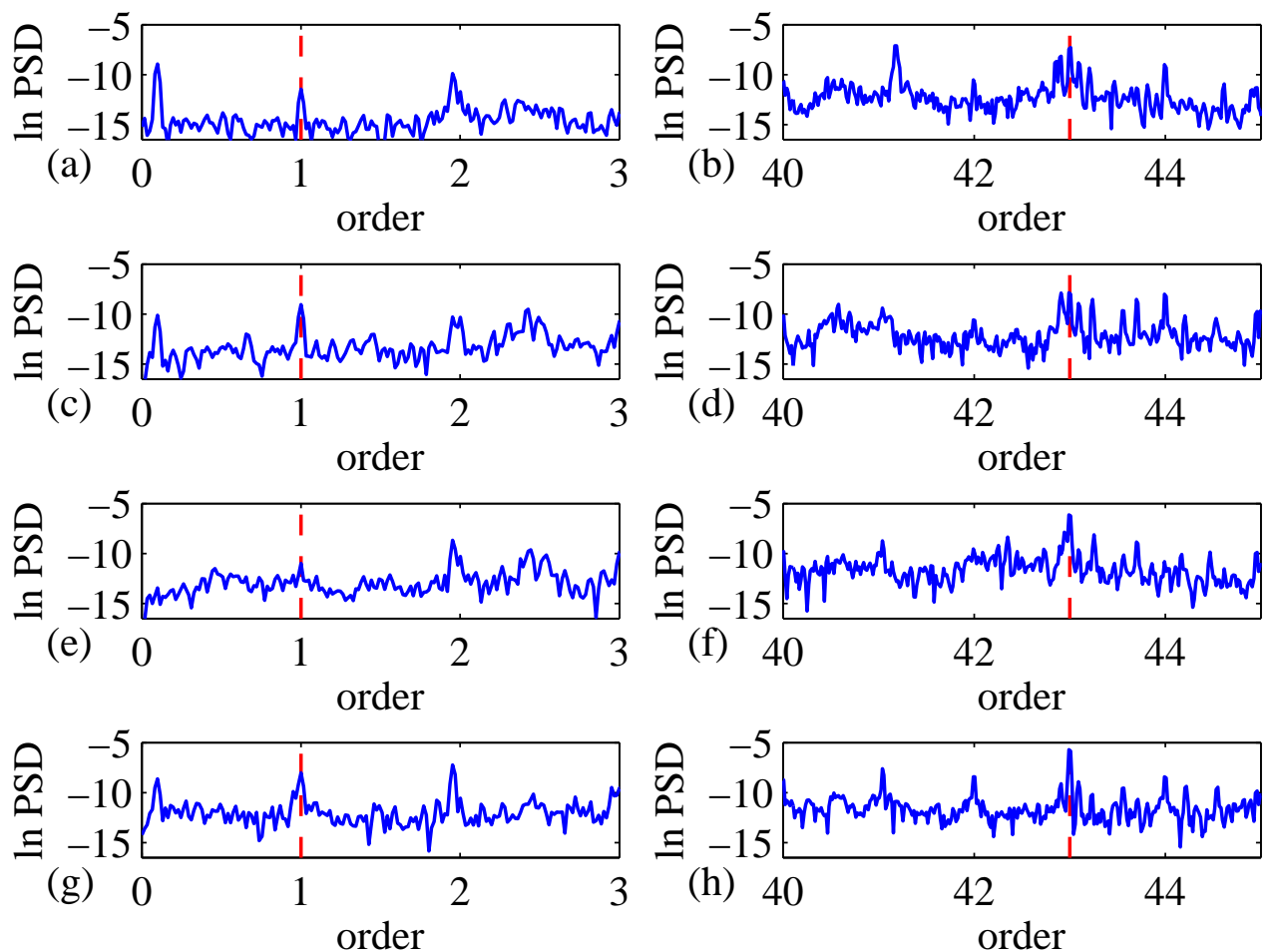

Figure 10: Close up of the natural logarithm of the order domain PSDs of the 0.5Hz sinusoidal time-varying load. Figures (a), (c), (e) and (g) magnifies the energy components in the vicinity of the 1st order (gear rotational frequency) for progressive stages of damage. Figures (b), (d), (f) and (h) magnifies the energy components in the vicinity of the 43st order (mesh frequency) for progressive stages of damage.

Figures 10 (a), (c), (e) and (g) illustrate the signal energy around the first order component which correspond to the gear rotational frequency, while figures 10 (b), (d), (f) and (h) illustrate the signal energy associated with the 43 order (gear mesh frequency).

It is interesting to note the increase in the energy at the tooth-meshing frequency from a fault on one tooth. Damage to one tooth normally does not increase the tooth-mesh component since this component represents the averaged energy associated with the meshing of all of the teeth. This increased energy must be due to some nonlinear effects. It will be seen in vibration signal synchronous average (figure 11) that the signal energy does indeed slightly increase at sections other than just the damaged gear tooth. This might potentially be due to torsional dynamics induced by the shaft flywheel. Figures 10 (f) and (h) indicate increased energy at the tooth-meshing energy, as well as increased energy at the 42th and 44th orders. This is indicative of amplitude modulation between the 1st order (gear rotational frequency) and the 43th order (meshing frequency). This result is in line with the fact that the damage is on the gear and not the pinion. The energy associated with the 1st order does not consistently increase as the gear tooth damage progresses.

\section{Synchronous averages of the resampled vibration signal}

The vibration signal synchronous averages are computed, both for the gear and the pinion. 
Each signal is approximately 30s long. With gear and pinion rotational frequencies of respectively $5.19 \mathrm{~Hz}$ and $10.12 \mathrm{~Hz}$ this results in 154 and 302 complete revolutions of respectively the gear and the pinion.
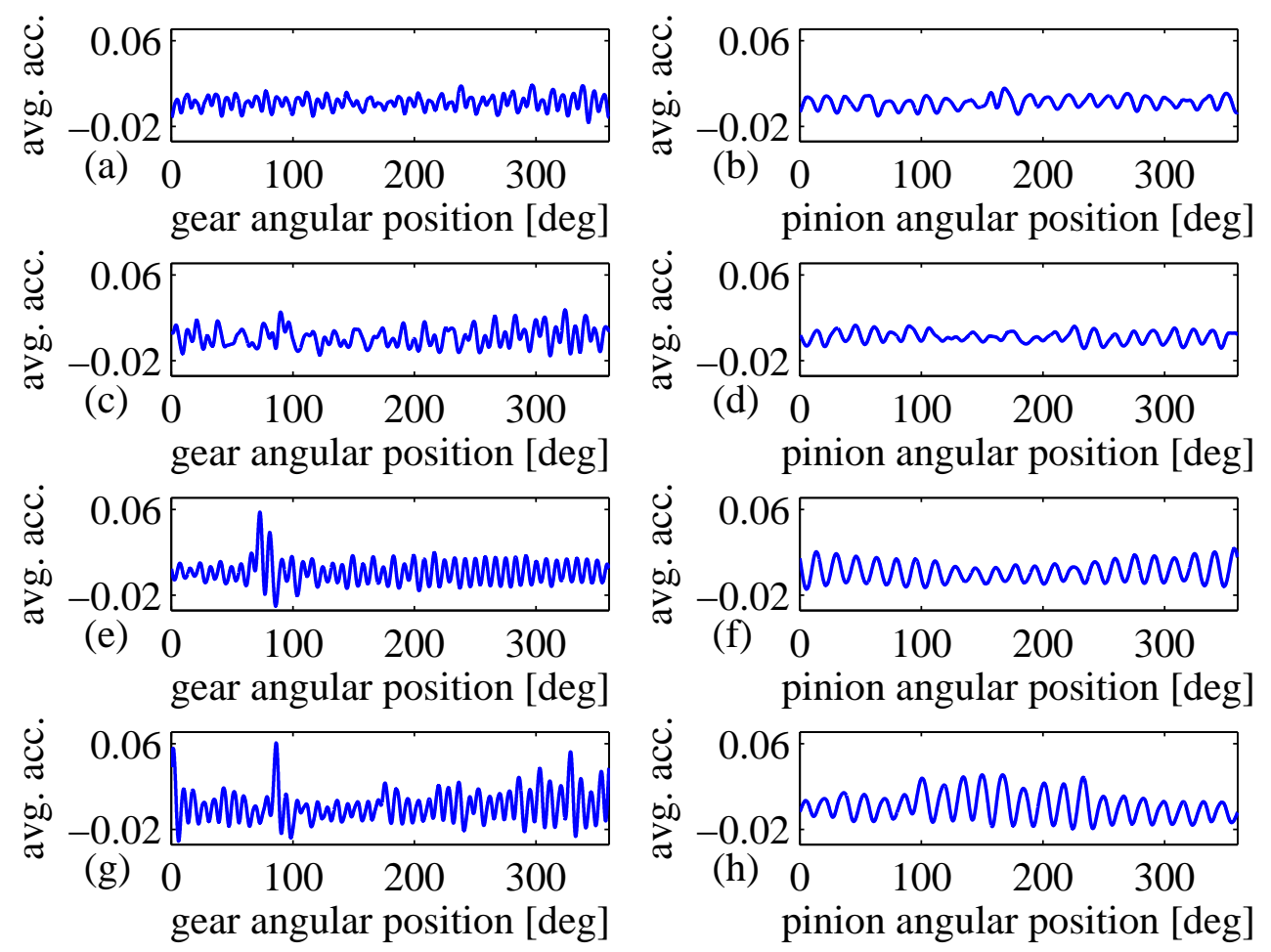

Figure 11: Vibration signal synchronous average for the $0.5 \mathrm{~Hz}$ fluctuating load. Progressive localised gear damage is indicated in (a) - (d), while (e) - (h) depict the synchronous averages of the fault-free pinion.

Figures 11 (a)-(h) illustrate the vibration signal synchronous averages for the $0.5 \mathrm{~Hz}$ sinusoidal time-varying loading condition. The figures (a), (c), (e) and (f) indicate the synchronous averages for progressive gear damage, while (b), (d), (f) and (h) are computed with respect to the pinion. Notice that the X-axis (angular position) indicated for the pinion is not consistent. This is because the tachometer is mounted on the gear shaft and not the pinion. The initial pinion angular phase is unknown at the beginning of each signal measurement. It is however possible to align the pinion synchronous averages by optimising their cross-correlation.

The initial gear damage (first stage) is not evident from the gear synchronous averages, however the more advanced second and third stages of damage is evident. The nature of the damage is however not readily evident. The pinion synchronous averages are also influenced by the presence of the gear tooth damage. This renders the pinion synchronous averages somewhat ambiguous, as it may appear that the pinion is subject to general wear.

\section{NLL discrepancy measure implementation}

The angular resampled frequency has 8600 datum points per revolution of the gear. The gear has 43 teeth, while the pinion has 22. A window length is selected so as to correspond to the gear meshing period. The window length is thus set equal to 200 datum points $\left(L_{w}=8600 / 43\right)$. 
A reference density distribution is estimated by means of a GMM with full-rank covariance matrices. The cross-validated NLL is used as measure of generalisation. It is found that 2 components generalise well. More components might be used without overfitting, but also without increased performance. It is also investigated whether conditioning the NLL on the gear angular speed improved the averaged magnitude of the NLL values on the validation set. Limited improvement is observed. It is subsequently decided to implement a 2 component GMM with full rank covariance matrices, without conditioning the NLL on any operating conditions.
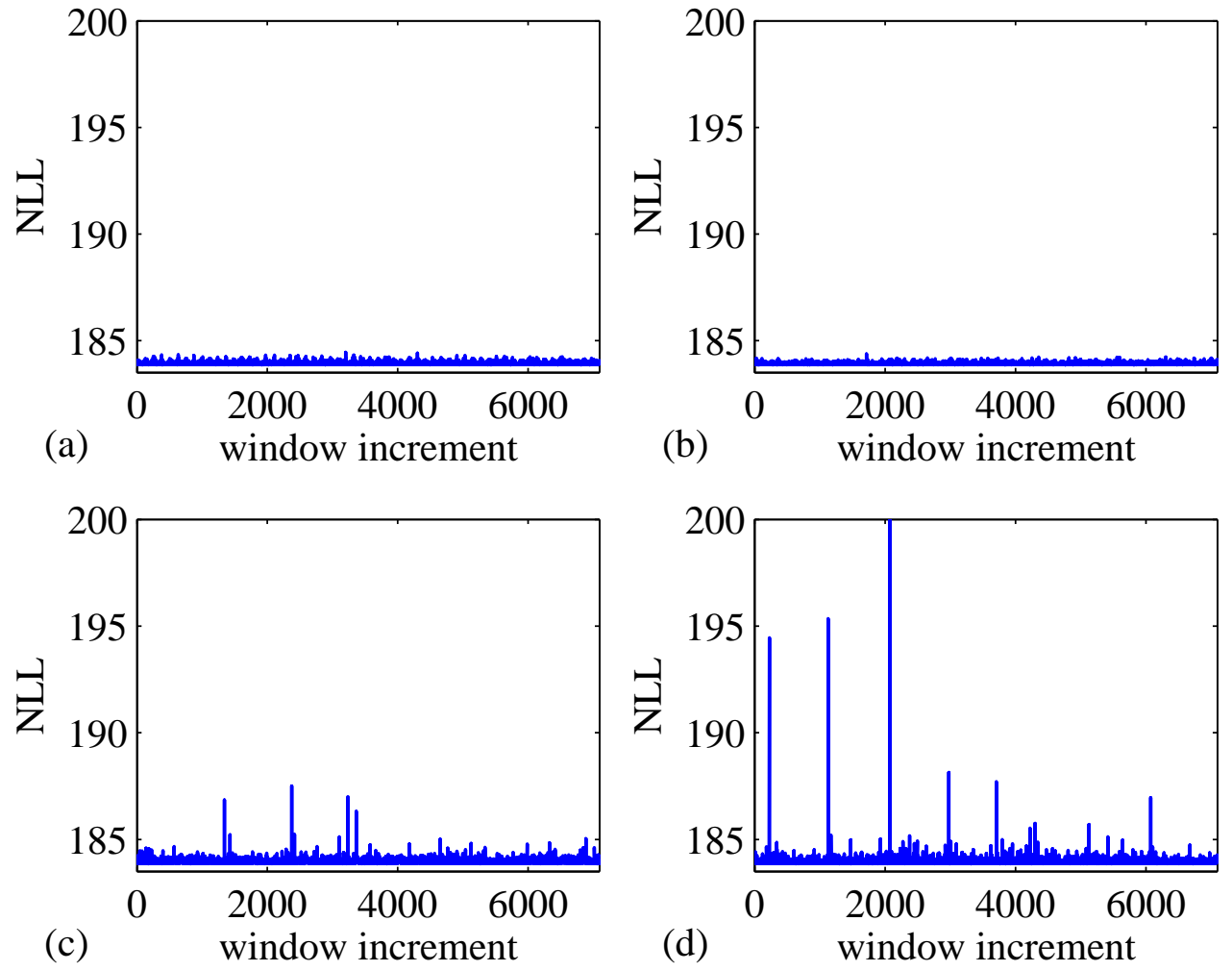

Figure 12: The NLL discrepancy signal associated with (a) the healthy gearbox, and (b)-(d) with increased stages of damage. The NLL discrepancy signal enhances the presence of fault induced signal outliers.

Figures 12 (a)-(d) illustrate the NLL discrepancy signal as computed for the $0.5 \mathrm{~Hz}$ sinusoidal loading condition. Figure 12 (a) illustrates the NLL values associated with the healthy gearbox, while (b)-(d) illustrate progressive stages of damage. The NLL discrepancy signal has a significantly lower sampling resolution (by a factor of 200) compared to the vibration signal. Each NLL datum point represents a single meshing period.

Figures 12 (a)-(d) illustrate the importance of analysing the natural logarithm of the likelihood, rather than the likelihood values themselves. The outliers must be scaled such that a few significant outliers do not completely dominate the discrepancy signal. The likelihood values of outliers in figures 12 (c) and (d) are so large (and subject to such variance) that the likelihood (compared to the log likelihood) synchronous averages would be very noisy and difficult to interpret. The NLL synchronous averages are also more linearly proportional to the magnitude of the fault induced damage. 

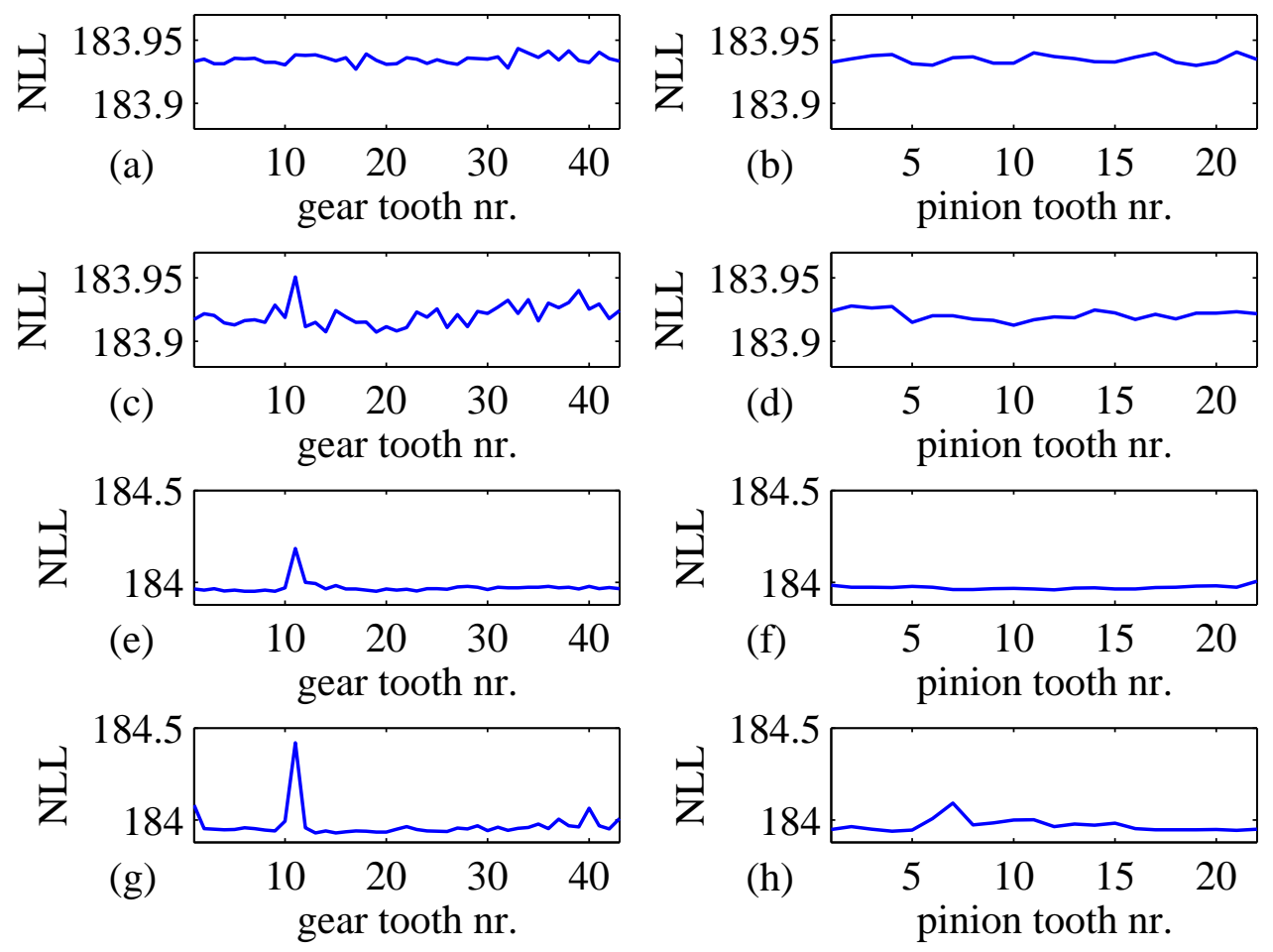

Figure 13: The NLL synchronous averages for the $0.5 \mathrm{~Hz}$ sinusoidal time-varying load condition, as computed with respect to the gear for progressive stages of damage (a), (c), (e) and (f), and as computed with respect to the pinion for progressive stages of wear (b), (d), (f) and (h). Please notice that the y-axes on (a)-(d) are scaled different to those of (e)-(h).

The NLL synchronous averages for the $0.5 \mathrm{~Hz}$ sinusoidal fluctuating operating as computed with respect to the gear for progressive stages of damage are depicted in figures 13 (a), (c), (e) and (f), and as computed with respect to the pinion for progressive stages of wear are depicted in (b), (d), (f) and (h). The initial (first stage) damage on the gear results in very small NLL values. For this reason the y-axes on figures 13 (a)-(d) are magnified compared to the later stages of damage depicted in (e)-(h). The damage condition is fairly simple to interpret and the presence of damage of the gear is quite evident.

The results obtained for the other loading conditions proved conceptually very similar to the presented results for the $0.5 \mathrm{~Hz}$ sinusoidal loading condition. Future research should be conducted to investigate the methodology under greater time-varying operating conditions.

\section{Conclusion}

This paper proposed a novel gear condition monitoring method which comprise two steps:

Firstly a NLL transform of a vibration signal is computed. The NLL transform is used to enhance diagnostic information in the signal, while suppressing signal components which are representative of normal operating 
conditions. The NLL is less sensitive to fluctuating load conditions, while also being smoother than the original signal.

Secondly the structure in the NLL discrepancy signal is analysed by means of synchronous averaging. The NLL synchronous average allows for an intuitive yet sensitive and robust, visual comparison of the novelty of signal patterns at different angular positions. The novelty of signal patterns can be used as an indication of the presence of damage.

A simple analytic example was used to illustrate how time-varying operating conditions (and especially phase modulation) may impede the ability of conventional vibration signal synchronous averaging to extract high frequency signal components. In this simple case study the NLL synchronous average proved significantly more sensitive to high frequency fault induced signal components.

Vibration data from an experimental gear test rig were subsequently used to further investigate the proposed methodology. An E20A Flender gearbox was subjected to a number of different time-varying loading conditions. Each of the loading conditions were used to generate vibration data for one of four stages of induced damage on a gear tooth. The NLL synchronous average proved simpler to interpret and more sensitive the induced fault compared to order domain spectral analysis and conventional synchronous averaging.

It is believed that the proposed methodology may be used to generate a simple, yet robust representation of the conditions of a gearbox. This representation may potentially be used to visualise the condition of a gearbox, or as a preprocessing stage to extract features which may subsequently be of use in a diagnostic change detection, or in a fault classification algorithm.

The research presented in this paper was conducted with the aim of finding an approach which may lead to a cost effective diagnostic technique for the condition monitoring of rotating machines. It is believed that the proposed methodology proves worthy of further investigation. Future research should especially consider the potential of the proposed methodology on more extensive time-varying operating conditions.

\section{Acknowledgement}

The authors gratefully acknowledge Dr. C.J. Stander for allowing access to, and providing support with the experimental data.

[1] S. Braun, The extraction of periodic waveforms by time domain averaging, Acustica 32 (1975) 69-77

[2] A.K.S. Jardine, D. Lin, D. Banjevic, A review on machinery diagnostics and prognostics implementing condition-based maintenance, Mechanical Systems and Signal Processing, Elsevier 20 (2006) 1483-1510

[3] E. Bechhoefer, M. Kingsley, A review of time synchronous average algorithms, Annual Conference of the Prognostics and Health Management Society (2009)

[4] S. Braun, The synchronous (time domain) average revisited, Mechanical Systems and Signal Processing 25 (2011) 1087-1102

[5] T. Barszcz, R.B. Randall, Application of spectral kurtosis for detection of a tooth crack in the planetary gear of a wind turbine, Mechanical Systems and Signal Processing 23 (2009) 1352-1365

[6] C.J. Stander, P.S. Heyns, Transmission path phase compensation for gear monitoring under fluctuating load conditions, Mechanical Systems and Signal Processing 20 (7) (2006) 1511-1522

[7] W. Wang, A.K. Wong, Autoregressive model-based gear fault diagnosis, Journal of Vibration and Acoustics, Transactions of the ASME 124 (2) (2002) 172-179 
[8] T. Heyns, S.J. Godsill, J.P. de Villiers, P.S. Heyns, Statistical gear health analysis which is robust to fluctuating loads and operating speeds, Mechanical Systems and Signal Processing, Transactions of the ASME 27 (2012) 651-666

[9] D. Miljković, Review of novelty detection methods, Information and Communication Technology, Electronics and Microelectronics, MIPRO Proceedings of the 33rd International Convention (2010) 593-598

[10] M. Timusk, M. Lipsett, C.K. Mechefske, Fault detection using transient machine signals, Mechanical Systems and Signal Processing 22 (7) (2008) 1724-1749

[11] A.S. Malegaonkar, A.M. Ariyaeeinia, P. Sivakumaran, Efficient speaker change detection using adapted Gaussian mixture model, IEEE Transactions on Audio, Speech, and Languadge Processing 15 (6) (2007) 1859-1869

[12] C.M. Bishop, SpringerLink (Online service), Pattern recognition and machine learning, Springer New York (2006)

[13] R.O. Duda, P.E. Hart, D.G. Stork, Pattern classification, Wiley (2001)

[14] N.H. Pontoppidan, J. Larsen, Unsupervised condition change detection in large diesel engines, Neural Networks for Signal Processing, IEEE XIII Workshop on (2003) 565-574

[15] P. Smyth, Model selection for probabilistic clustering using cross-validated likelihood, Statistics and Computing 9 (2000) 63-72

[16] P.D. McFadden, Determining the location of a fatigue crack in a gear from the phase of the change in the meshing vibration, Mechanical Systems and Signal Processing 2 (4) (1988) 403-409

[17] F. Combet, L. Gelman, An automated methodology for performing time synchronous averaging of a gearbox signal without speed sensor, Mechanical Systems and Signal Processing 21 (6) (2007) 2590-2606

[18] C.J. Stander, P.S. Heyns, Instantaneous angular speed monitoring of gearboxes under non-cyclic stationary load conditions, Mechanical Systems and Signal Processing 19 (4) (2005) 817-835 